\title{
Laparoscopic colon resection with intraoperative polyp localisation with high resolution ultrasonography coupled with colour power Doppler
}

\author{
F Panaro, M Casaccia, D Cavaliere, P Torelli
}

Postgrad Med J 2003;79:533-534

A 40 year old woman with a $3 \mathrm{~cm}$ sigmoid polyp lesion who underwent a laparoscopic colon resection after intraoperative localisation of the lesion using laparoscopic ultrasonography coupled with colour power Doppler is described. She has successful intraoperative detection of the polyp followed by radical laparoscopic removal of the lesion. The advantage of using laparoscopic high resolution ultrasonography coupled with colour power Doppler to locate colonic polyp lesions during a laparoscopic colon resection is that intraoperative colonoscopy can be avoided. Intraoperative ultrasonography of the colon can accurately localise colonic polyp lesions that are not detectable during laparoscopy and represents a quick and effective alternative to other imaging techniques.

p ntraoperative laparoscopic ultrasonography to locate colonic polyp lesions during a laparoscopic colon resection has several advantages: it is easy, fast, and highly accurate in localising intraoperatively the presence of a lesion otherwise nonpalpable during laparoscopy. In our experience it does not require the instillation of normal saline to increase the sonographic enhancement of the surrounding structures. A lack of adverse effects and short examination time are some advantages of this method. However, adequate training for surgeons unfamiliar with this technology is recommended.

We describe a successful intraoperative detection of a polyp in a 40 year old woman with high resolution ultrasonography using a flexible tip ultrasound probe coupled with colour power Doppler followed by radical laparoscopic removal of the lesion.

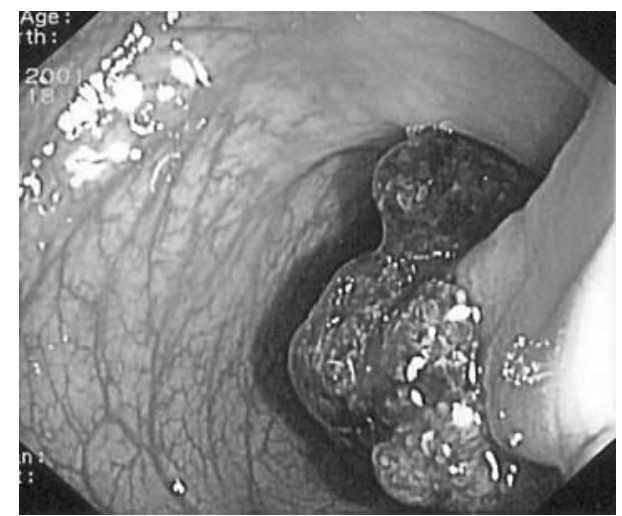

Figure 1 Colonoscopy shows the polyp lesion at the level of the sigmoid colon.

\section{CASE REPORT}

A 40 year old woman (body mass index 21\%) was admitted for a single episode of rectal bleeding. Double contrast enema revealed a $3 \mathrm{~cm}$ polyp at the level of sigma. An endoscopic extirpation of the polyp was attempted but not completed because of its large base of implantation. Biopsy during colonoscopy (fig l) revealed a focal adenocarcinoma in situ, $30 \mathrm{~cm}$ from the anal verge. The patient was than referred for surgery: a laparoscopic approach was chosen with the patient placed in the supine position; four ports (one $10 \mathrm{~mm}$, one $12 \mathrm{~mm}$, and two $5 \mathrm{~mm}$ ) were placed in the lower abdomen in a diamond fashion. Intraoperative ultrasound was performed using a 7.5 $\mathrm{MHz}$ flexible tip probe with colour power Doppler placed through the $12 \mathrm{~mm}$ port. The probe was directed in the operative field under direct laparoscopic viewing. The left colon was scanned from the rectosigmoid junction to the splenic flexure, focusing at the sigma region. The polyp was easily identified as an isohypoechoic image (fig 2) with a vascular structure at colour power Doppler. The level of the lesion was then marked with a clip on the serosal surface to facilitate its localisation. The left colon was then mobilised and exteriorised through a protected abdominal incision at the left iliac fossa trocar site. Sigmoid resection and lymphatic dissection was performed, with respect of the oncological principles. Bowel continuity was restored through an end-to-end manual anastomosis. Operative time was 62 minutes. No peroperative complications occurred. Postoperative hospital stay was characterised by return of bowel peristalsis on postoperative day 1 , resumption of oral feeding on postoperative day 2 , and discharge on postoperative day 3. Recovery was uneventful. Histological examination confirmed the malignant nature of the lesion (polyp

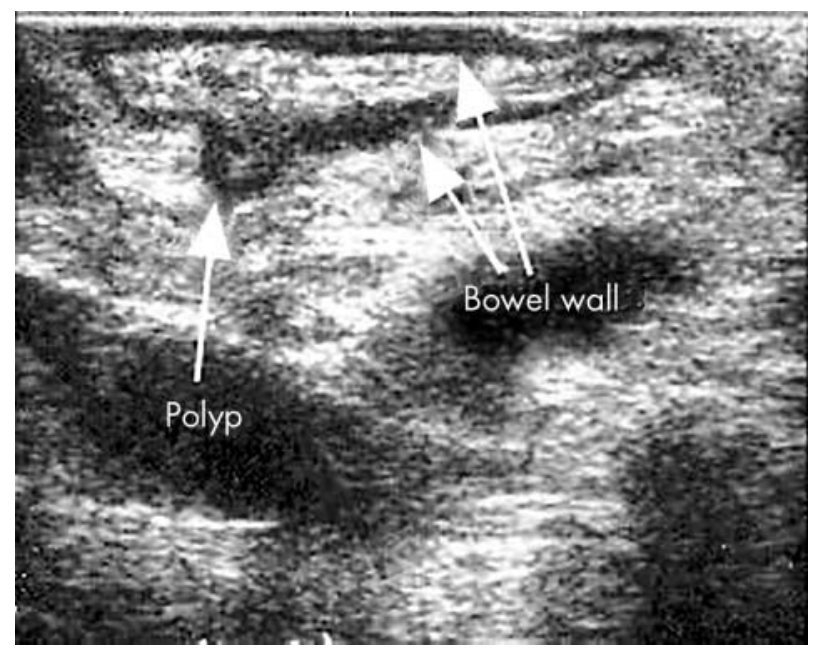

Figure 2 Intraoperative ultrasound shows the sigmoid polyp enhanced by intraluminal bowel gas. 
with adenocarcinoma in situ, grade II) and margins of resection free of neoplastic infiltration. Twelve lymph nodes were identified in the surgical specimen without any sign of metastatic invasion.

\section{DISCUSSION}

The most common strategies now used to localise colonic polyp lesions include preoperative detection by contrast enema, ${ }^{1}$ intraoperative colonoscopy, ${ }^{2}$ and marking of the colonic wall adjacent to the lesion with activated carbon ${ }^{3}$ or India ink ${ }^{4}$ during preoperative colonoscopy. Recent reports have suggested laparoscopic intracorporeal ultrasonography to be a useful imaging technique in abdominal surgery. ${ }^{5-8}$ Intraoperative colonic polyp localisation using intracorporeal ultrasound has been described only recently. ${ }^{90}$ However, this technique requires instillation of sterile normal saline into the colon and rectum. We showed that detection of polyp lesions that were not detectable during laparoscopy, using the most up-to-date ultrasonographic intraoperative instrument (high resolution 7.5 MHz flexible tip probe with colour power Doppler), does not require intracolonic or extracolonic, intrabdominal, saline instillation as a means of hyperechoic enhancement but only the natural intraluminal bowel gas. The colour power Doppler feature of the ultrasonographic probe also allowed us to identify the presence of a vascular structure in the polyp, thus increasing the sensitivity and the specificity of the procedure. A disadvantage is that adequate training for surgeons is necessary to optimise its efficacy.

\section{CONCLUSION}

Intraoperative high resolution ultrasonography with colour power Doppler of the colon without intraluminal or extraluminal instillation of sterile normal saline is feasible, noninvasive, and highly accurate in localising a polyp lesion. It is a quick, efficient alternative to other common strategies used to localise colonic polyp lesions.

\section{Authors' affiliations}

F Panaro, University of Illinois at Chicago, Chicago, Illinois, USA M Casaccia, D Cavaliere, P Torelli, Department of Organ Transplant, St Martino Hospital, University of Genoa, Advanced Laparoscopic Unit, Genoa, Italy

Correspondence to: Dr F Panaro, University of Illinois at Chicago, College of Medicine, 840 South Wood Street (room 402), Chicago, IL 60612-7322, USA; fpanaro@uic.edu

Submitted 23 January 2003

Accepted 6 March 2003

\section{REFERENCES}

1 Lord SA, Larach SW, Ferrara A, et al. Laparoscopic resection for colorectal cancer: a three-year experience. Dis Colon Rectum 1996;39: 148-54

2 Sakanove Y, Nakao K, Shoji Y, et al. Intraoperative colonoscopy. Surg Endosc 1993;7:84-7.

3 Kitamura K, Yamane T, Oyama T, et al. Rapid and accurate method for delineating cancer lesions in laparoscopic colectomy using activated carbon injection. J Surg Oncol 1995;58: 1-34.

4 Gellman L, Salky B, Edye M. Laparoscopic assisted colectomy. Surg Endosc 1996;10:1041-4.

5 Catheline JM. Laparoscopic ultrasonography of the liver and the biliary tract. J Chir 2002;3:160-4.

6 Lavonius MI, Laine S, Salo S, et al. Role of laparoscopy and laparoscopic ultrasound in staging of pancreatic tumours. Ann Chir Gynaecol 2001:4:252-5

7 Pautler SE, Choyke PL, Pavlovich CP, et al. Intraoperative ultrasound aids in dissection during laparoscopic partial adrenalectomy. J Urol 2002; 168(4 pt 1):1352-5.

8 Flett ME, Lim MN, Bruce D, et al. Prognostic value of laparoscopic ultrasound in patients with gastro-esophageal cancer. Dis Esophagus $2001 ; 3-4: 223-6$

9 Luck AJ, Thomas ML, Roediger WEW, et al. Localization of the nonpalpable colonic lesion with intraoperative ultrasound. Surg Endosc 1999;5:526-7.

10 Grazia E, Targarona EM, Garrige J, et al. Laparoscopic treatment of colorectal polyps. Gastroenterol Hepatol 2000;23:224-7. 